\title{
Exact Simulation of Gamma-driven Ornstein-Uhlenbeck Processes with Finite and Infinite Activity Jumps*
}

\author{
Yan $\mathrm{Qu}^{\dagger}$ \\ Angelos Dassios ${ }^{\ddagger}$ \\ London School of Economics \\ London School of Economics \\ Hongbiao Zhao ${ }^{\S}$ \\ Shanghai University of Finance and Economics
}

9th August 2019

\begin{abstract}
We develop a distributional decomposition approach for exactly simulating two types of Gamma-driven Ornstein-Uhlenbeck (OU) processes with time-varying marginal distributions: the Gamma-OU process and the OU-Gamma process. The former has finite-activity jumps, and its marginal distribution is asymptotically Gamma; the latter has infinite-activity jumps that are driven by a Gamma process. We prove that the transition distributions of the two processes at any given time can be exactly decomposed into simple elements: at any given time, the former is equal in distribution to the sum of one deterministic trend and one compound Poisson random variable (r.v.); the latter is equal in distribution to the sum of one deterministic trend, one compound Poisson r.v., and one Gamma r.v.. The results immediately lead to very efficient algorithms for their exact simulations without numerical inversion. Extensive numerical experiments are reported to demonstrate the accuracy and efficiency of our algorithms.
\end{abstract}

Keywords: Monte Carlo simulation; Exact simulation; Non-Gaussian Ornstein-Uhlenbeck (OU) process; GammaOU process; OU-Gamma process; Shot-noise process; Finite activity; Infinite activity; Gamma process

Mathematics Subject Classification (2010): Primary: 65C05 - 62E15 - 68U20; Secondary: 60G51 60 H35 $60 J 75$

\footnotetext{
* An edited version to be published by Journal of the Operational Research Society

${ }^{\dagger}$ Department of Statistics, London School of Economics, Houghton Street, London WC2A 2AE, United Kingdom. Email: y.qu3@1se.ac.uk

${ }^{\ddagger}$ Department of Statistics, London School of Economics, Houghton Street, London WC2A 2AE, United Kingdom. Email: a.dassios@1se.ac.uk

${ }^{\S}$ Corresponding author, School of Statistics and Management, Shanghai University of Finance and Economics, No. 777 Guoding Road, Shanghai 200433, China; Shanghai Institute of International Finance and Economics, No. 777 Guoding Road, Shanghai 200433, China. Email: h.zhao1@lse.ac.uk
} 


\section{Introduction}

The classical Gaussian Ornstein-Uhlenbeck (OU) process was first introduced by Uhlenbeck and Ornstein (1930) in physics, and later became popular in finance, economics and many other fields. For example, Vasicek (1977) adopted the OU process to describe the evolution of interest rates. This seminal work in finance was followed by a variety of extensions, with one very important example being the family of non-Gaussian OU processes, see Barndorff-Nielsen $(1997,1998)$, Barndorff-Nielsen et al. (1998), and Barndorff-Nielsen and Shephard (2001b, 2002). The idea is to replace the original Brownian motion in the OU process by a general Lévy process. The resulting stochastic process retains the property of mean-reverting dynamics, and also possesses some stylised features such as jumps and skewness, which often form the essential components of real financial data.

Naturally, one candidate Lévy process for replacing Brownian motion in the OU process is a Gamma process. Gamma process ${ }^{1}$ is simple and highly mathematically tractable, which has been used as a very popular representative for Lévy processes in the literature, see Barndorff-Nielsen and Shephard (2001a,b, 2003a), Schoutens (2003), Cont and Tankov (2004), Kyprianou (2006) and Schoutens and Cariboni (2010). This type of OU process driven by a Gamma process is named as the $O U$-Gamma $(O U-\Gamma)$ process in Barndorff-Nielsen and Shephard (2003a). The OU- $\Gamma$ process has been used to model stochastic volatilities (Barndorff-Nielsen and Shephard, 2003a; Brockwell et al., 2007; Granelli and Veraart, 2016), human mortality rates, actuarial valuations (Hainaut and Devolder, 2008) and instantaneous short rates of interest (Norberg, 2004; Eberlein et al., 2013). A closely related counterpart is the so-called Gamma-OU (Г-OU) process ${ }^{2}$, which is linked to a Gamma variate in a very different way. It is an OU process who has a Gamma marginal distribution. Barndorff-Nielsen and Shephard (2001b, 2002, 2003a,b) used the $\Gamma$-OU process to model the stochastic volatility of stock prices, see also Roberts et al. (2004), Jongbloed et al. (2005), Griffin and Steel (2006), Creal (2008) and Frühwirth-Schnatter and Sögner (2009). Moreover, Nicolato and Venardos (2003) further applied the $\Gamma$-OU stochastic volatility model to pricing European options. Schoutens and Cariboni (2010) and Bianchi and Fabozzi (2015) adopted the $\Gamma$-OU process as a stochastic intensity process for modelling credit default risk and pricing credit default swaps (CDSs). Cartea et al. (2015, p.220) used it for modelling the stochastic mean-reverting volume rate of trading, see also Cartea and Jaimungal (2016).

\footnotetext{
${ }^{1}$ It is fundamentally built upon a Gamma distribution. As an important alternative to the normal distribution, the Gamma distribution is widely used as the building block for a variety of useful stochastic processes, such as the Gamma process and variance Gamma process, see Madan and Seneta (1990) and Madan et al. (1998).

${ }^{2}$ The abbreviation "Г-OU" is borrowed from Barndorff-Nielsen and Shephard (2003a).
} 
As concluded by Barndorff-Nielsen (1997), the $\Gamma-\mathrm{OU}$ and OU- $\Gamma$ processes are very tractable models that could facilitate many potential applications. Despite the similar names, they are very different pure-jump processes. The former has finite-activity jumps (i.e., finite jumps over any finite time horizon), whereas the latter has infinite-activity jumps (i.e., infinite jumps over any finite time horizon). Using our simulation algorithms developed later in this paper, their key difference can be clearly observed in the plots of several sample paths in Figure 1, where the parameters are set such that they all have the same initial levels and expected levels at any time. Finite-activity jumps can be very useful for capturing large and infrequent shocks, whereas infinite-activity jumps are better suited to small and frequent shocks. In real financial data, both types may exist. Recently, AïtSahalia and Jacod $(2009,2011)$ found that high-frequency stock price data present infinite-activity jumps. Ornthanalai (2014) also provided evidence for infinite-activity jumps in index options and returns from 1996 to 2009, and suggested that infinite-activity jumps, instead of the Brownian increments, should be the default modelling choice in asset pricing models.

In this paper, our focus is on the development of exact simulation algorithms for the two types of Gamma-driven OU processes with time-varying marginal distributions. The exact simulation scheme has the primary advantage of generating the sample paths according to the process law exactly without bias (Broadie and Kaya, 2006; Chen and Huang, 2013). We design efficient algorithms mainly based on the principle of exact distributional decomposition. Without using any numerical inverse transform, we discover a strategy whereby the $\Gamma$-OU and $\mathrm{OU}-\Gamma$ processes can be directly decomposed into several basic elements: the former has one deterministic trend and one compound Poisson random variable (r.v.), whereas the latter has one deterministic trend, one compound Poisson r.v., and one Gamma r.v. Each element can be simulated directly or using existing packages (such as the Gamma variate generator). We conduct extensive numerical experiments and demonstrate the accuracy and efficiency of our algorithms. In particular, for the $\Gamma$-OU process, there exists a straightforward alternative exact scheme, i.e., a path-dependent approach that is trivially based on its definition as a shot-noise process (2.2). We carry out a numerical comparison with our new decomposition scheme. Note that this simple path-dependent approach is not applicable for the OU- $\Gamma$ process because of its infinite activity. Recently, Zhang and Zhang (2008, 2009), Zhang (2011) and Kawai and Masuda (2011a,b, 2012) developed simulation algorithms for Gamma-driven OU processes with stationary marginal distributions rather than time-varying marginal distributions. The mythologies for their algorithm designs are mainly based on the well known Lévy-Khintchine representation for infinitely divisible distributions such as Gamma distribution and tempered stable (TS) distribution. Apparently it is applicable, as the marginal distributions of their processes are simply time-invariant and infinitely divisible distributions under the key 

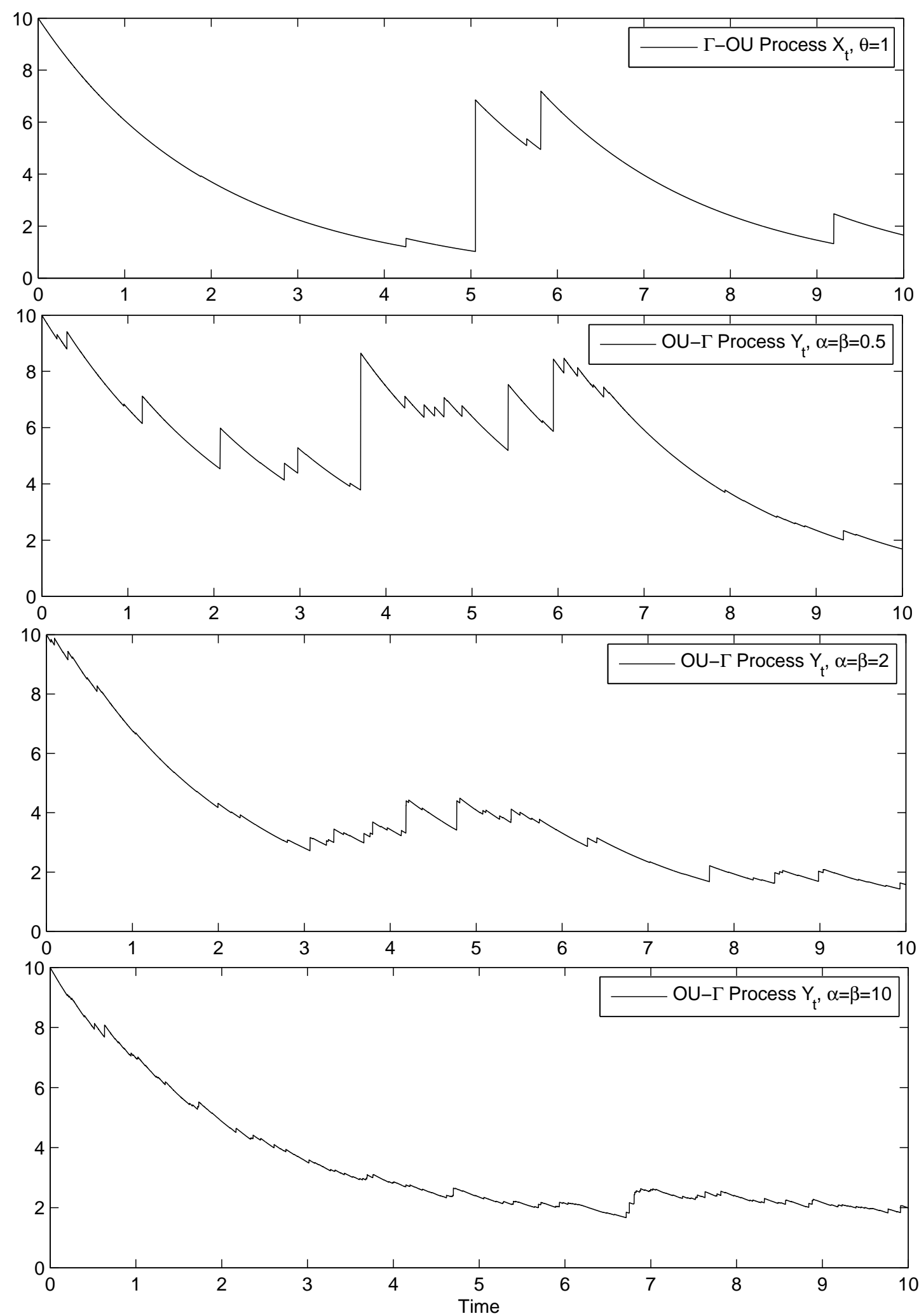

Figure 1: Simulated sample paths of one finite-activity $\Gamma$-OU process $X_{t}$ (Algorithm 3.1) and three infiniteactivity OU- $\Gamma$ processes $Y_{t}$ (Algorithm 4.1) in the period $t \in[0,10]$, with the same initial level $X_{0}=Y_{0}$ and expected level $\mathbb{E}\left[X_{t} \mid X_{0}\right]=\mathbb{E}\left[Y_{t} \mid Y_{0}\right]$ based on the parameters $\left(\delta, \rho ; \theta ; X_{0}\right)=(0.5,1.0 ; 1.0 ; 10.0),\left(\delta, \varrho ; \alpha, \beta ; Y_{0}\right)=(0.5,1.0 ; 0.5,0.5 ; 10.0),\left(\delta, \varrho ; \alpha, \beta ; Y_{0}\right)=$ $(0.5,1.0 ; 2.0,2.0 ; 10.0)$, and $\left(\delta, \varrho ; \alpha, \beta ; Y_{0}\right)=(0.5,1.0 ; 10.0,10.0 ; 10.0)$, respectively. 
assumption of stationarity for their processes due to the change of time. However, in will become clear later that Lévy-Khintchine representation is not applicable to our processes whose marginal distributions are time-varying and more importantly are not necessarily infinitely divisible. In fact, our approach is different and it is mainly based on the exact distributional decomposition approach, which has also been adopted recently by Dassios and Zhao (2013, 2017), Dassios et al. (2018) and Qu et al. (2019) for exactly sampling some popular point processes and random variables.

The remainder of this paper is organised as follows: Section 2 offers the preliminaries including formal mathematical definitions and introductions for OU-Gamma (OU- $\Gamma)$ process and $\Gamma-\mathrm{OU}$ process, respectively. Section 3 and Section 4 present some theoretical results for Laplace transforms and the exact simulation algorithms for the $\Gamma-\mathrm{OU}$ and $\mathrm{OU}-\Gamma$ processes, respectively. In Section 5 , we describe extensive numerical experiments and demonstrate the accuracy and efficiency of our algorithms. In particular, for the $\Gamma$-OU case, a numerical comparison between the traditional path-dependent scheme and our newly developed scheme is provided. Section 6 concludes this paper and suggests some possible extensions as topics for further research.

\section{Preliminaries}

The $O U$-Gamma $(O U$ - $\Gamma)$ process $Y=\left\{Y_{t}, t \geq 0\right\}$ is a positive stochastic process on the positive half-line $\mathbb{R}_{+}$following the stochastic differential equation

$$
\mathrm{d} Y_{t}=-\delta Y_{t} \mathrm{~d} t+\varrho \mathrm{d} Z_{t}, \quad t \geq 0,
$$

where

- $\delta>0$ is the mean-reverting (or exponential decay) rate;

- $\varrho>0$ is a positive constant;

- $Z_{t}$ is a Gamma process with shape parameter $\alpha>0$ and rate parameter $\beta>0$, i.e., $Z_{1} \sim$ $\Gamma(\alpha, \beta)$.

Given the the initial level $Y_{0}>0$, it is alternatively defined by

$$
Y_{t}=Y_{0} e^{-\delta t}+\varrho \int_{0}^{t} e^{-\delta(t-s)} \mathrm{d} Z_{s}, \quad t \geq 0 .
$$

Here, $Z_{t}$ is generally called the background driving Lévy process (BDLP) (Barndorff-Nielsen and Shephard, 2001b, 2002). If $Z_{t}$ is replaced by a standard Brownian motion, then $Y_{t}$ becomes the 
ordinary Gaussian OU process (Uhlenbeck and Ornstein, 1930).

The Gamma-OU ( $\Gamma-O U)$ process $X=\left\{X_{t}, t \geq 0\right\}$ is a positive stochastic process on the positive half-line $\mathbb{R}_{+}$following the stochastic differential equation

$$
\mathrm{d} X_{t}=-\delta X_{t} \mathrm{~d} t+\mathrm{d} Z_{t}, \quad t \geq 0
$$

where $Z_{t}$ is a compound Poisson process of rate $\rho>0$, and the jump sizes follows an exponential distribution with rate parameter $\theta>0$.

Given the initial level $X_{0}>0$, it is alternatively defined by

$$
X_{t}=X_{0} e^{-\delta t}+\sum_{0 \leq T_{i}<t} Z_{i} e^{-\delta\left(t-T_{i}\right)}, \quad t \geq 0,
$$

where

- $\left\{Z_{i}\right\}_{i=1,2, \ldots}$ is a sequence of independent, identically distributed (i.i.d.) jump sizes, each of which follows an exponential distribution with rate parameter $\theta$, i.e., $Z_{i} \sim \operatorname{Exp}(\theta)$ for any $i$;

- $\left\{T_{i}\right\}_{i=1,2, \ldots}$ are the arrival times of a standard Poisson process $N_{t}$ with constant rate $\rho$.

Note that, the initial values $X_{0}$ and $Y_{0}$ can be either deterministic or random. This key difference between the two processes $X_{t}$ and $Y_{t}$ above is that, the $\Gamma$-OU process has finite-activity jumps (i.e., finite jumps over any finite time horizon), as the Lévy density of a Gamma process has an infinite integral, and the process has infinitely many jumps over any time interval, whereas the OU- $\Gamma$ process has infinite-activity jumps (i.e., infinite jumps over any finite time horizon), as it can essentially be considered as a Poisson shot-noise process with exponentially distributed jump sizes as pointed out by Barndorff-Nielsen and Shephard (2001b, p.175), Roberts et al. (2004) and Griffin and Steel (2006). It can be clearly observed in the simulated sample paths in Figure 1, where the parameters are set such that they all have the same initial levels and expected levels at any time, i.e., $X_{0}=Y_{0}$ and $\mathbb{E}\left[X_{t} \mid X_{0}\right]=\mathbb{E}\left[Y_{t} \mid Y_{0}\right]$.

Note that, our processes belong to the original Lévy-driven OU processes, which have also been considered by Wolfe (1982), Sato and Yamazato (1984), Barndorff-Nielsen (1998), BarndorffNielsen et al. (1998), Jongbloed et al. (2005), Masuda and Yoshida (2005), Brockwell et al. (2007) and Mai (2014), and have also been applied in finance by Barndorff-Nielsen and Shephard (2003a), Norberg (2004), Hainaut and Devolder (2008), Eberlein et al. (2013) and Granelli and Veraart (2016). They are different from the modified (time-changed) Lévy-driven OU processes used in 
Zhang and Zhang (2008, 2009), Zhang (2011), Kawai and Masuda (2011a,b, 2012) and Bianchi et al. (2017), where there is an unusual time change in the BDLP, i.e.,

$$
\mathrm{d} Y_{t}=-\delta Y_{t} \mathrm{~d} t+\varrho \mathrm{d} Z_{\delta t}, \quad t \geq 0 .
$$

In fact, our Lévy-driven OU processes are directly constructed upon the original $B D L P Z_{t}$ rather than the time-changed BDLP $Z_{\delta t}$ in (2.3). The differences between these two types can also be found in Barndorff-Nielsen (1998); Barndorff-Nielsen and Shephard (2003a) and Schoutens (2003, p.48). Their key difference is that, the marginal distributions of time-changed Lévy-driven OU processes as defined by Barndorff-Nielsen and Shephard (2001b, p.168) are very restrictive, which are invariant (time-homogenous) and have to specifically follow stationary laws. However, the initial values of our processes, $X_{0}$ and $Y_{0}$, are much more flexible, which can be either deterministic or random, so the marginal distributions are time-varying in general.

\section{Exact Simulation of Gamma-OU Process}

Let us start by looking at the simpler of the two Gamma-driven OU processes, i.e., the $\Gamma$-OU process $X_{t}$ as defined by (2.2). It can be exactly simulated via the distributional decomposition approach, which can be identified from the following conditional Laplace transform ${ }^{3}$.

Theorem 3.1. The Laplace transform of $X_{t+\tau}$ conditional on $X_{t}$ is given by

$$
\mathbb{E}\left[e^{-v X_{t+\tau}} \mid X_{t}\right]=e^{-v w X_{t}} \times \exp \left(-\frac{\rho}{\delta} \int_{0}^{\infty}\left(1-e^{-v s}\right) \int_{1}^{\frac{1}{w}} \theta u e^{-\theta u s} \frac{1}{u} \mathrm{~d} u \mathrm{~d} s\right), \quad v \in \mathbb{R}^{+},
$$

where $\tau>0$ is any fixed-length time interval and $w:=e^{-\delta \tau}$.

Proof. Based on the Laplace transform of the Poisson shot-noise process provided in Corollary 2.4 of Dassios and Jang (2003, p.79), we have

$$
\mathbb{E}\left[e^{-v X_{t+\tau}} \mid X_{t}\right]=e^{-v w X_{t}} \exp \left(-\rho \int_{t}^{t+\tau}\left[1-\hat{h}_{Z_{i}}\left(v e^{-\delta(t+\tau-s)}\right)\right] \mathrm{d} s\right)
$$

where $\hat{h}_{Z_{i}}(\cdot)$ is the Laplace transform of jump sizes. The jump sizes are assumed to follow an

\footnotetext{
${ }^{3}$ When working with non-negative random variables, it is common for a lot of researchers to use Laplace transforms rather than characteristic functions, see Bertoin (1998, Chapter III), Bertoin (1999), Sato (1999, Chapter 6) and Norberg (2004). In fact, all of our calculations can be repeated using characteristic functions instead, and we avoid potential complications with complex arguments.
} 
exponential distribution with rate $\theta>0$. Then, we have a simple Laplace transform

$$
\hat{h}_{Z_{i}}(u)=\frac{\theta}{\theta+u} .
$$

Rewriting the integral term in (3.2) as

$$
\begin{aligned}
\int_{t}^{t+\tau}\left[1-\hat{h}_{Z_{i}}\left(v e^{-\delta(t+\tau-s)}\right)\right] \mathrm{d} s & =\int_{0}^{\tau}\left[1-\hat{h}_{Z_{i}}\left(v e^{-\delta x}\right)\right] \mathrm{d} x \\
& =\frac{1}{\delta} \int_{v w}^{v} \frac{1-\hat{h}_{Z_{i}}(u)}{u} \mathrm{~d} u \\
& =\frac{1}{\delta} \int_{v w}^{v} \frac{1}{u} \int_{0}^{\infty}\left(1-e^{-u y}\right) \theta e^{-\theta y} \mathrm{~d} y \mathrm{~d} u \\
& =\frac{1}{\delta} \int_{0}^{\infty} \frac{\left(1-e^{-v s}\right)}{s} \int_{s}^{\frac{s}{w}} \theta e^{-\theta y} \mathrm{~d} y \mathrm{~d} s \\
& =\frac{1}{\delta} \int_{0}^{\infty}\left(1-e^{-v s}\right) \frac{e^{-\theta s}-e^{-\theta \frac{s}{w}}}{s} \mathrm{~d} s \\
& =\frac{1}{\delta} \int_{0}^{\infty}\left(1-e^{-v s}\right) \int_{\theta}^{\frac{\theta}{w}} e^{-s u} \mathrm{~d} u \mathrm{~d} s \\
& =\frac{1}{\delta} \int_{0}^{\infty}\left(1-e^{-v s}\right) \int_{1}^{\frac{1}{w}} \theta u e^{-s \theta u} \frac{1}{u} \mathrm{~d} u \mathrm{~d} s
\end{aligned}
$$

the conditional Laplace transform of $X_{t}$ can be expressed as (3.1).

The Laplace transform of $X_{t+\tau}$ conditional on $X_{t}$ in Theorem 3.1 implies that $X_{t+\tau}$ has two simple elements, one deterministic trend and one compound Poisson r.v. This result immediately leads to an exact simulation algorithm.

Algorithm 3.1 (Exact Simulation of $\Gamma$-OU Process via Decomposition Approach). The distribution of $X_{t+\tau}$ conditional on $X_{t}$ can be exactly decomposed as

$$
X_{t+\tau} \mid X_{t} \stackrel{\mathcal{D}}{=} \underbrace{e^{-\delta \tau} X_{t}}_{\text {Deterministic trend }}+\underbrace{\sum_{k=1}^{N} S_{k},}_{\text {Finite jumps }} \quad \tau \in \mathbb{R}^{+},
$$

where

- $N$ is a Poisson r.v. of rate $\rho \tau$; 
- $\left\{S_{k}\right\}_{k=1,2, \ldots}$ are i.i.d. r.v.s following a mixture of exponential distributions, i.e.,

$$
S_{k} \sim \operatorname{Exp}\left(\theta e^{\delta \tau U}\right), \quad U \sim \mathcal{U}[0,1], \quad \forall k=1,2, \ldots
$$

It is well known that there exists a simple alternative algorithm. This is a path-dependent approach that is constructed directly based on the definition of the $\Gamma$-OU process as a Poisson shot-noise process (2.2).

Algorithm 3.2 (Exact Simulation of $\Gamma$-OU Process via Path-dependent Approach). Given the $i^{\text {th }}$ jump arrival time $T_{i}$ and the associated level $X_{T_{i}}$, we can exactly simulate the next arrival time $T_{i+1}$ and the associated level $X_{T_{i+1}}$ by the following steps:

1. Generate an exponentially distributed $r . v . \tau_{i+1}^{*} \sim \operatorname{Exp}(\rho)$ as the $(i+1)^{\text {th }}$ inter-arrival time;

2. Record the next jump arrival time $T_{i+1}=T_{i}+\tau_{i+1}^{*}$;

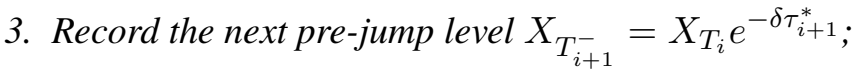

4. Generate an exponentially distributed r.v. $Z_{i+1} \sim \operatorname{Exp}(\theta)$ as the $(i+1)^{\text {th }}$ jump size;

5. Record the next level $X_{T_{i+1}}=X_{T_{i+1}^{-}}+Z_{i+1}$.

An obvious advantage of our decomposition approach (Algorithm 3.1) over the traditional pathdependent approach (Algorithm 3.2) is that it can generate the distribution directly at the target terminal $t+\tau$ without drawing complete skeletons of the underlying paths from the initial time $t$ to the terminal $t+\tau$. This key methodological difference between the two approaches is illustrated in Figure 2, where the simulation starts at time 0. Later, in Section 5, we will perform a detailed numerical comparison.

Another advantage of our decomposition approach is that it can be further extended to develop an exact simulation algorithm for the OU- $\Gamma$ process, to which the path-dependent approach is not applicable.

\section{Exact Simulation of OU-Gamma Process}

Let us first derive the conditional Laplace transform of the OU- $\Gamma$ process.

Theorem 4.1. The Laplace transform of $Y_{t+\tau}$ conditional on $Y_{t}$ can be expressed as

$$
\mathbb{E}\left[e^{-v Y_{t+\tau}} \mid Y_{t}\right]
$$




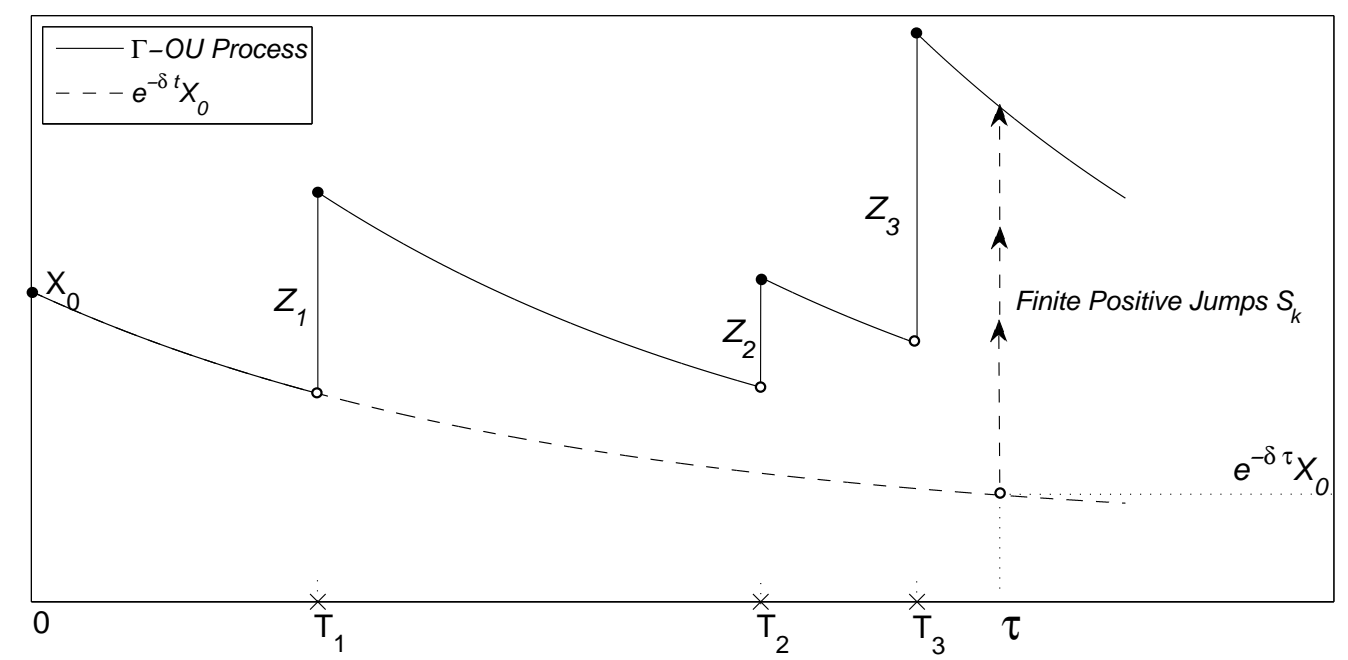

Figure 2: Illustration of the path-dependent approach of Algorithm 3.2 and the decomposition approach of Algorithm 3.1 for simulating the distribution of $X_{\tau}$ given the initial level $X_{0}$ at time 0 .

$$
\begin{aligned}
= & e^{-v w Y_{t}} \times \exp \left(-\frac{\alpha \varrho}{\delta} \ln w \int_{0}^{\infty}\left(1-e^{-v s}\right) s^{-1} e^{-\frac{\beta}{w} s} \mathrm{~d} s\right) \\
& \times \exp \left(-\frac{\alpha \varrho}{\delta} \int_{0}^{\infty}\left(1-e^{-v s}\right) \int_{1}^{\frac{1}{w}} \beta e^{-\beta u s} \ln u \mathrm{~d} u \mathrm{~d} s\right), \quad v \in \mathbb{R}^{+} .
\end{aligned}
$$

Proof. The OU- $\Gamma$ process $Y_{t}$ in (2.1) is driven by a Gamma process $Z_{t}$ with Laplace exponent

$$
\Phi(v)=\alpha \ln \left(1+\frac{v}{\beta}\right), \quad v \in \mathbb{R}^{+}
$$

and Lévy measure

$$
\nu(\mathrm{d} s)=\alpha s^{-1} e^{-\beta s} \mathrm{~d} s, \quad s \geq 0 .
$$

It can be considered as the limit of a compound Poisson process (Madan and Seneta, 1990), i.e.,

$$
Z_{t}=\lim _{\epsilon \downarrow 0} \sum_{i=1}^{M_{t}} Z_{i}^{\epsilon}
$$

where

- $M_{t}$ is a standard Poisson process of rate $\frac{1}{\epsilon}, \epsilon>0$;

- $\left\{Z_{i}^{\epsilon}\right\}_{i=1,2 \ldots}$ are i.i.d. jump sizes with density

$$
\mathrm{d} H_{Z_{i}^{\epsilon}}(y)=\frac{\beta^{\alpha \epsilon}}{\Gamma(\alpha \epsilon)} y^{\alpha \epsilon-1} e^{-\beta y} \mathrm{~d} y
$$


where $\Gamma(\cdot)$ is the gamma function, i.e., $\Gamma(u):=\int_{0}^{\infty} s^{u-1} e^{-s} \mathrm{~d} s$.

This implies that the OU- $\Gamma$ process $Y_{t}$ is the limit of a Poisson shot-noise process with Gammadistributed jump sizes, i.e., $Y_{t}=\lim _{\epsilon \downarrow 0} Y_{t}^{\epsilon}$, where

$$
Y_{t}^{\epsilon}=e^{-\delta t} Y_{0}+\varrho \sum_{0 \leq T_{i}^{*}<t} e^{-\delta\left(t-T_{i}^{*}\right)} Z_{i}^{\epsilon}
$$

and $\left\{T_{i}^{*}\right\}_{i=1,2, \ldots}$ is the sequence of event arrival times of the Poisson process $M_{t}$. In fact, $Y_{t}^{\epsilon}$ is a Markov process. By the piecewise-deterministic Markov processes theory (Davis, 1984), the generator of $\left(Y_{t}^{\epsilon}, t\right)$ acting on any function $f(y, t)$ within its domain $\Omega\left(\mathcal{A}_{\epsilon}\right)$ is given by

$$
\mathcal{A}_{\epsilon} f(y, t)=\frac{\partial f}{\partial t}-\delta y \frac{\partial f}{\partial y}+\frac{\varrho}{\epsilon}\left[\int_{0}^{\infty} f(y+z, t) \mathrm{d} H_{Z_{i}^{\epsilon}}(z)-f(y, t)\right] .
$$

Using the martingale approach ${ }^{4}$, we can easily find a suitable martingale by setting $\mathcal{A}_{\epsilon} f(y, t)=0$, and then obtain

$$
\mathbb{E}\left[e^{-v Y_{t+\tau}^{\epsilon}} \mid Y_{t}^{\epsilon}\right]=e^{-v w Y_{t}^{\epsilon}} \exp \left(-\varrho \int_{t}^{t+\tau} \frac{1-\hat{h}_{Z_{i}^{\epsilon}}\left(v e^{-\delta(t+\tau-s)}\right)}{\epsilon} \mathrm{d} s\right),
$$

where

$$
\hat{h}_{Z_{i}^{\epsilon}}(u)=\int_{0}^{\infty} e^{-u z} \mathrm{~d} H_{Z_{i}^{\epsilon}}(z)=\left(1+\frac{u}{\beta}\right)^{-\epsilon \alpha} .
$$

Furthermore, by the dominated convergence theorem, we have

$$
\begin{aligned}
\mathbb{E}\left[e^{-v Y_{t+\tau}} \mid Y_{t}\right] & =\lim _{\epsilon \downarrow 0} \mathbb{E}\left[e^{-v Y_{t+\tau}^{\epsilon}} \mid Y_{t}^{\epsilon}\right] \\
& =e^{-v w Y_{t}} \exp \left(-\varrho \int_{t}^{t+\tau} \lim _{\epsilon \downarrow 0} \frac{1-\left[1+\frac{v}{\beta} e^{-\delta(t+\tau-s)}\right]^{-\epsilon \alpha}}{\epsilon} \mathrm{d} s\right) \\
& =e^{-v w Y_{t}} \exp \left(-\varrho \int_{t}^{t+\tau} \lim _{\epsilon \downarrow 0} \frac{1-\exp \left(-\epsilon \alpha \ln \left[1+\frac{v}{\beta} e^{-\delta(t+\tau-s)}\right]\right)}{\epsilon} \mathrm{d} s\right) \\
& =e^{-v w Y_{t}} \exp \left(-\alpha \varrho \int_{t}^{t+\tau} \ln \left[1+\frac{v}{\beta} e^{-\delta(t+\tau-s)}\right] \mathrm{d} s\right) .
\end{aligned}
$$

According to (4.3) and (4.5), we can further rewrite the conditional Laplace transform as

$$
\mathbb{E}\left[e^{-v Y_{t+\tau}} \mid Y_{t}\right]=e^{-v w Y_{t}} \exp \left(-\frac{\varrho}{\delta} \int_{v w}^{v} \frac{\alpha}{u} \ln \left(1+\frac{u}{\beta}\right) \mathrm{d} u\right)
$$

${ }^{4}$ For details of the martingale approach, see Dassios and Embrechts (1989), Dassios and Jang (2003), and Dassios and Zhao (2011). 


$$
=e^{-v w Y_{t}} \exp \left(-\frac{\varrho}{\delta} \int_{v w}^{v} \frac{1}{u} \int_{0}^{\infty}\left(1-e^{-u y}\right) \alpha y^{-1} e^{-\beta y} \mathrm{~d} y \mathrm{~d} u\right),
$$

where

$$
\begin{aligned}
& \int_{v w}^{v} \frac{1}{u} \int_{0}^{\infty}\left(1-e^{-u y}\right) \alpha y^{-1} e^{-\beta y} \mathrm{~d} y \mathrm{~d} u \\
= & \int_{0}^{\infty} \frac{1-e^{-v s}}{s} \int_{s}^{\frac{s}{w}} \alpha y^{-1} e^{-\beta y} \mathrm{~d} y \mathrm{~d} s \\
= & \int_{0}^{\infty} \frac{1-e^{-v s}}{s} \int_{s}^{\frac{s}{w}} \alpha y^{-1} e^{-\beta \frac{s}{w}} \mathrm{~d} y \mathrm{~d} s+\int_{0}^{\infty} \frac{1-e^{-v s}}{s} \int_{s}^{\frac{s}{w}} \alpha y^{-1}\left(e^{-\beta y}-e^{-\beta \frac{s}{w}}\right) \mathrm{d} y \mathrm{~d} s .
\end{aligned}
$$

Note that, both of the terms in (4.7) are positive, because $e^{-\beta y} \geq e^{-\beta \frac{s}{w}}$ for any $y \in\left[s, \frac{s}{w}\right]$.

1. The first term of (4.7) can be expressed as the Laplace exponent of a Gamma r.v., i.e.,

$$
\int_{0}^{\infty} \frac{1-e^{-v s}}{s} \int_{s}^{\frac{s}{w}} \alpha y^{-1} e^{-\beta \frac{s}{w}} \mathrm{~d} y \mathrm{~d} s=\alpha \ln \left(\frac{1}{w}\right) \int_{0}^{\infty}\left(1-e^{-v s}\right) s^{-1} e^{-\frac{\beta}{w} s} \mathrm{~d} s .
$$

2. The inner integral of the second term of (4.7) can be rewritten as

$$
\begin{aligned}
& \frac{1}{s} \int_{s}^{\frac{s}{w}} \alpha y^{-1}\left(e^{-\beta y}-e^{-\beta \frac{s}{w}}\right) \mathrm{d} y \\
= & \alpha \int_{1}^{\frac{1}{w}} \frac{1}{x} \frac{e^{-\beta s x}-e^{-\beta \frac{s}{w}}}{s} \mathrm{~d} x \\
= & \alpha \int_{1}^{\frac{1}{w}} x^{-1} \int_{x}^{\frac{1}{w}} \beta e^{-\beta s u} \mathrm{~d} u \mathrm{~d} x \\
= & \alpha \int_{1}^{\frac{1}{w}} \beta e^{-\beta s u} \ln u \mathrm{~d} u .
\end{aligned}
$$

Finally, we can obtain the conditional Laplace transform (4.1) based on the integral representations of (4.8) and (4.9).

The distribution of $Y_{t+\tau}$ conditional on $Y_{t}$ can be exactly decomposed into three basic components: one deterministic trend, one Gamma r.v., and one compound Poisson r.v.

Algorithm 4.1 (Exact Simulation of OU- $\Gamma$ Process). The distribution of $Y_{t+\tau}$ conditional on $Y_{t}$ 
can be exactly decomposed as

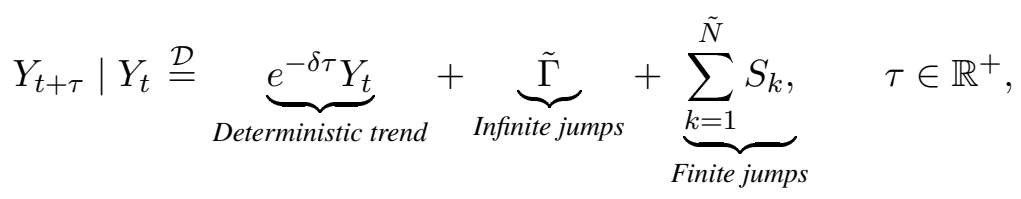

where

- $\tilde{\Gamma}$ is a Gamma r.v. of

$$
\tilde{\Gamma} \sim \Gamma\left(\alpha \varrho \tau, \beta e^{\delta \tau}\right)
$$

- $\tilde{N}$ is a Poisson r.v. of rate $\frac{1}{2} \alpha \varrho \delta \tau^{2}$;

- $\left\{S_{k}\right\}_{k=1,2, \ldots}$ are i.i.d. r.v.s. following a mixture of exponential distributions, i.e.,

$$
S_{k} \sim \operatorname{Exp}\left(\beta e^{\delta \tau \sqrt{U}}\right), \quad U \sim \mathcal{U}[0,1], \quad \forall k=1,2, \ldots
$$

Proof. The three components of (4.10) correspond to the three terms of the conditional Laplace transform (4.1), respectively:

1. The first term of (4.1) is the Laplace transform of $e^{-\delta \tau} Y_{t}$.

2. The second term of (4.1) is the Laplace transform of a Gamma r.v., $\tilde{\Gamma}$, and the corresponding Lévy measure is specified by

$$
\nu(\mathrm{d} s)=\frac{\alpha \varrho}{\delta} \ln \left(\frac{1}{w}\right) s^{-1} e^{-\frac{\beta}{w} s} \mathrm{~d} s .
$$

3. The Laplace exponent of the third term in (4.1) can be rewritten as

$$
\frac{\alpha \varrho}{\delta} \int_{0}^{\infty}\left(1-e^{-v s}\right) \int_{1}^{\frac{1}{w}} \beta e^{-\beta u s} \ln u \mathrm{~d} u \mathrm{~d} s=\frac{\alpha \varrho}{2 \delta} \ln ^{2} w \int_{0}^{\infty}\left(1-e^{-v s}\right) \int_{1}^{\frac{1}{w}} \beta u e^{-\beta u s} f_{V}(u) \mathrm{d} u \mathrm{~d} s,
$$

where

$$
f_{V}(u)=\frac{2}{\ln ^{2} w} \frac{\ln u}{u}, \quad u \in\left[1, \frac{1}{w}\right]
$$

is the density function of r.v. $V$. This clearly indicates that (4.13) is the Laplace exponent of a compound Poisson r.v. whose rate parameter is $\frac{\alpha \varrho}{2 \delta} \ln ^{2} w$ and jump sizes are exponentially distributed with rate $\beta V$. Here, $V$ is a well-defined r.v. that can be exactly simulated via an explicit inverse transform, as the cumulative distribution function (CDF) of $V$, i.e.,

$$
F_{V}(u)=\left(\frac{\ln u}{\ln w}\right)^{2}, \quad u \in\left[1, \frac{1}{w}\right]
$$


can be inverted explicitly as

$$
F_{V}^{-1}(x)=w^{-\sqrt{x}}, \quad x \in[0,1]
$$

For the decomposition specified by (4.10), the compound Poisson r.v. $\sum_{k=1}^{\tilde{N}} S_{k}$ can only produce a finite number of (large) jumps. In contrast with the decomposition in (3.3) for the $\Gamma$-OU process, the extra term of the Gamma r.v. $\tilde{\Gamma}$ in (4.10) clearly explains why the OU- $\Gamma$ process has infiniteactivity jumps for any time interval. That is, within any time interval $\tau>0$, there always exists a Gamma r.v. $\tilde{\Gamma}$ that produces an infinite number of (small) positive jumps.

\section{Simulation Studies}

In this section, we illustrate the performance and effectiveness of our algorithms through extensive numerical experiments. The simulations were conducted on a desktop computer with an Intel Core i7-6700 CPU @3.40 GHz, 24.00 GB RAM, and Windows 10 Professional 64-bit operating system. The algorithms were coded and performed in MatLab (R2012a), and the computation time was measured by the elapsed CPU time in seconds. The numerical validation and tests for our algorithms are based on the true means (5.1) and (5.2) for the $\Gamma-\mathrm{OU}$ and $\mathrm{OU}-\Gamma$ processes, respectively. The associated errors with respect to the true values are reported by three standard measures:

1. Error $=$ estimated value - true value;

2. Relative error (error \%) $=\frac{\text { estimated value }- \text { true value }}{\text { true value }}$

3. Root mean square error $\mathrm{RMSE}=\sqrt{\mathrm{bias}^{2}+\mathrm{SE}^{2}}$, where $\mathrm{SE}$ is the standard error of the simulation output, and the bias is the difference between the expectation of the estimator and the associated true (theoretical) value; for the exact simulation algorithm, the bias is conventionally set to zero.

The theoretical means at any given time $T>0$ for the two processes are as follows:

- For the $\Gamma$-OU process $X_{t}$,

$$
\mathbb{E}\left[X_{T} \mid X_{0}\right]=e^{-\delta T} X_{0}+\frac{\rho}{\delta}\left(1-e^{-\delta T}\right) \frac{1}{\theta}
$$


- For the OU- $\Gamma$ process $Y_{t}$,

$$
\mathbb{E}\left[Y_{T} \mid Y_{0}\right]=e^{-\delta T} Y_{0}+\frac{\varrho}{\delta}\left(1-e^{-\delta T}\right) \frac{\alpha}{\beta}
$$

In the numerical implementations, the parameters were set as follows:

- $\left(\delta, \rho ; \theta ; X_{0}\right)=(0.5,1.0 ; 1.0 ; 10.0)$ for the $\Gamma$-OU process $X_{t}$;

- $\left(\delta, \varrho ; \alpha, \beta ; Y_{0}\right)=(0.5,1.0 ; 2.0,2.0 ; 10.0)$ for the OU- $\Gamma$ process $Y_{t}$.

The means of (5.1) and (5.2) in simple analytic forms are used to numerically validate our algorithms. In fact, mean provides us the most convenient way to test and verify newly-developed algorithms, as the true value of mean can be easily derived in a simple analytic form as above. Of course, other higher moments or some values of probabilities also can be used for testing as long as they have analytic forms so we have already known the true values exactly. For example, the conditional Laplace transforms $\mathbb{E}\left[e^{-v X_{t+\tau}} \mid X_{t}\right]$ and $\mathbb{E}\left[e^{-v Y_{t+\tau}} \mid Y_{t}\right]$ that we have derived in Theorem 3.1 and Theorem 4.1 could be used for testing as well. However, it would be better for us to further derive their forms more analytically, otherwise, we would have to discretize and truncate the infinite integrals in the Laplace transforms, which would introduce estimation errors. Basically, the means can be tested by a sufficient number of different parameter choices, the aim of testing and verifying our algorithms numerically can be achieved very similarly based on the simple mean and more complicated moments, so we choose means for simplicity and it also avoid additional estimation errors. Our tests and validations based on the means have been carried out based a vast number of various different parameter sets, and the results based on other parameter sets show very similar levels of accuracy and efficiency. To save space, we select representative examples in this paper.

The parameters here are selected for the purpose of validating our simulation algorithms only, and they are not estimated or calibrated from empirical work. In practical pricing applications, the parameters under the risk-neutral probability measure would be calibrated from market prices or rates as in Schoutens and Cariboni (2010) and Bianchi and Fabozzi (2015). In risk management applications, the parameters the natural probability measure would be estimated from historical time series as in Mai (2014). Large banks risk management systems use pricing functions to compute the value of derivatives portfolios: these systems usually consider both risk-neutral and historical information. Additionally, portfolio allocation strategies may consider risk-neutral and historical "data", see Bianchi and Tassinari (2018). Risk neutral and historical information are the two different faces of the same coin. 
Table 1: Comparison between the true means (5.1) and the associated simulation results using Algorithm 3.2 vs. Algorithm 3.1 for the $\Gamma$-OU process based on the parameters $\left(\delta, \rho ; \theta ; X_{0}\right)=(0.5,1.0 ; 1.0 ; 10.0)$ for time intervals $\tau=0.5,1$, respectively, with the associated plots provided in Figure 3.

\begin{tabular}{|c|c|c|c|c|c|c|c|c|c|c|c|}
\hline Interval $\tau$ & Paths & True & imulation & Error & Error \% & RMSE & Time & Simulation & Error & Error \% & $\overline{\text { RMSE Time }}$ \\
\hline & & & \multicolumn{5}{|c|}{ Algo. 3.2} & \multicolumn{4}{|c|}{ Algo. 3.1 } \\
\hline \multirow[t]{6}{*}{$\tau=0.5$} & 10,000 & 8.2304 & 8.2525 & 0.0221 & $0.27 \%$ & 0.0091 & 0.97 & 8.2222 & -0.0082 & $-0.10 \%$ & $0.0087 \quad 0.38$ \\
\hline & 40,000 & 8.2304 & 8.2398 & 0.0094 & $0.11 \%$ & 0.0045 & 3.64 & 8.2291 & -0.0013 & $-0.02 \%$ & $0.0044 \quad 1.39$ \\
\hline & 160,000 & 8.2304 & 8.2329 & 0.0025 & $0.03 \%$ & 0.0022 & 14.33 & 8.2288 & -0.0016 & $-0.02 \%$ & $0.0022 \quad 5.50$ \\
\hline & 640,000 & 8.2304 & 8.2314 & 0.0010 & $0.01 \%$ & 0.0011 & 59.48 & 8.2306 & 0.0002 & $0.00 \%$ & 0.001121 .92 \\
\hline & $2,560,000$ & 8.2304 & 8.2301 & -0.0003 & $0.00 \%$ & 0.0006 & 224.03 & 8.2304 & 0.0000 & $0.00 \%$ & 0.000688 .48 \\
\hline & & & \multicolumn{5}{|c|}{ Algo. 3.2 } & \multicolumn{4}{|c|}{ Algo. 3.1 } \\
\hline \multirow[t]{5}{*}{$\tau=1$} & 10,000 & 6.8522 & 6.8621 & 0.0099 & $0.14 \%$ & 0.0111 & 0.91 & 6.8418 & -0.0104 & $-0.15 \%$ & 0.01140 .38 \\
\hline & 40,000 & 6.8522 & 6.8559 & 0.0037 & $0.05 \%$ & 0.0056 & 3.61 & 6.8543 & 0.0021 & $0.03 \%$ & $0.0057 \quad 1.59$ \\
\hline & 160,000 & 6.8522 & 6.8535 & 0.0012 & $0.02 \%$ & 0.0028 & 14.11 & 6.8536 & 0.0014 & $0.02 \%$ & $0.0028 \quad 5.91$ \\
\hline & 640,000 & 6.8522 & 6.8504 & -0.0019 & $-0.03 \%$ & 0.0014 & 55.88 & 6.8515 & -0.0007 & $-0.01 \%$ & 0.001423 .19 \\
\hline & $2,560,000$ & 6.8522 & 6.8521 & -0.0001 & $0.00 \%$ & 0.0007 & 240.42 & 6.8522 & -0.0000 & $0.00 \%$ & 0.000792 .81 \\
\hline
\end{tabular}

For the $\Gamma$-OU case, we first conducted a numerical comparison between our decomposition scheme (Algorithm 3.1) and the traditional path-dependent scheme (Algorithm 3.2) for time intervals $\tau=0.5,1$. The detailed numerical results are reported in Table 3, where we can see that, for a slightly large number of paths, both algorithms are extremely accurate (in terms of error, error\%, and RMSE) by comparing the simulation-estimated means with the associated true values provided by (5.1). Moreover, the associated plots for the convergence analysis of RMSE vs. CPU time are presented in Figure 3, where we can clearly observe that our new scheme outperforms the traditional approach.

To simulate the $\Gamma$-OU and OU- $\Gamma$ processes using our decomposition scheme, we can also split the target time period $[0, T]$ into $n_{\tau}:=T / \tau$ smaller pieces and generate the sample paths in a piecewise manner ${ }^{5}$. Here, $n_{\tau}$ is the total number of equally-spaced discretized time steps. The four sample paths in Figure 1 were plotted using $n_{\tau}=10,000$. We set different values of $n_{\tau}$ to investigate how Algorithm 3.1 and Algorithm 4.1 perform for $T=1,2,4,10$ and $n_{\tau}=1,2,4,10$, respectively. The associated numerical results are reported in Table 2 and Table 3, and plots of their convergence are presented in Figure 4 and Figure 5, respectively, for the $\Gamma$-OU and OU- $\Gamma$ processes. From the two figures, we can observe an interesting pattern: the case $n_{\tau}=1$ is usually the best performer when the sample path is relatively short. However, when the sample path becomes longer, this is not always the optimal discretization. For instance, $n_{\tau}=2$ is the best performer for $T=10$ in Figure 4.

The numerical results reported here show that the simulations are very fast and the associated errors (measured in terms of error, error\%, and RMSE) are small. Overall, it is evident that our newly developed decomposition approach can achieve high accuracy as well as efficiency.

\footnotetext{
${ }^{5}$ Our algorithms are also applicable to any irregularly spaced intervals.
} 

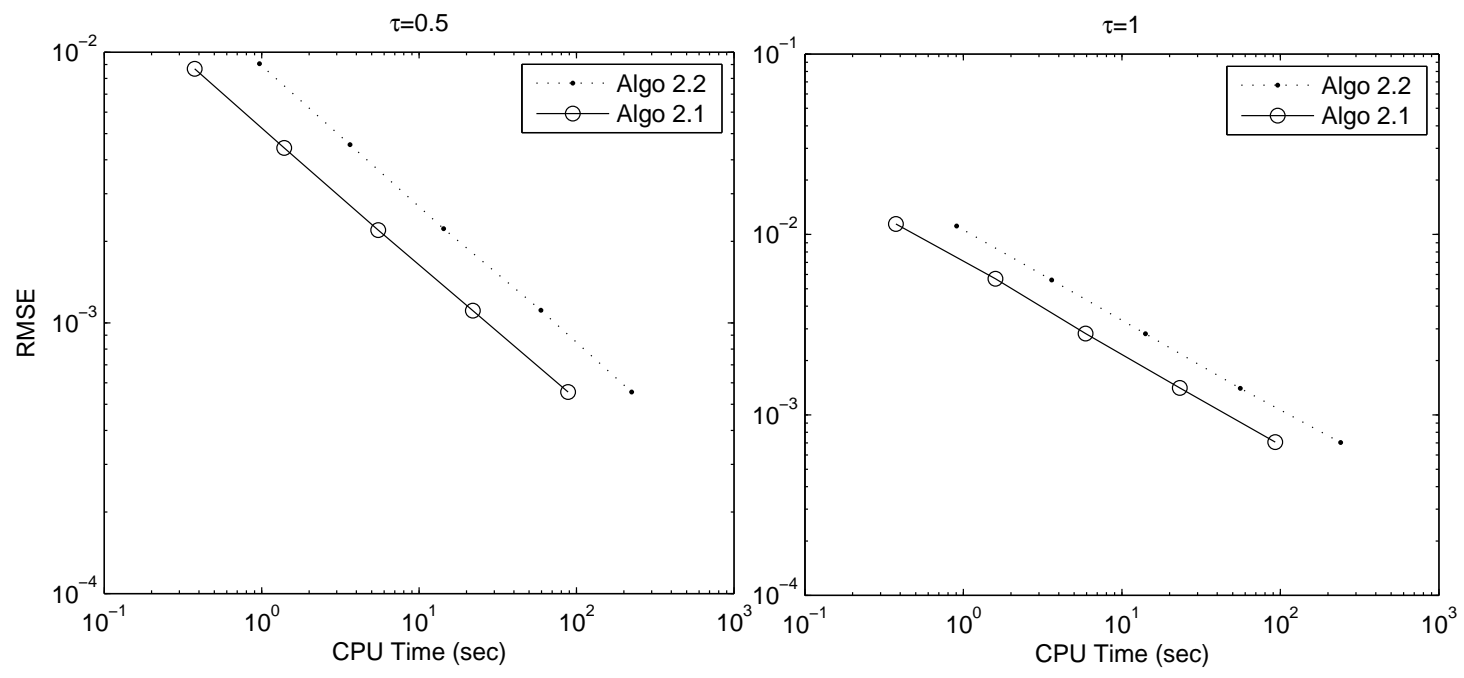

Figure 3: Convergence analysis of Algorithm 3.2 vs. Algorithm 3.1 for the $\Gamma$-OU process $X_{t}$ based on the parameters $\left(\delta, \rho ; \theta ; X_{0}\right)=(0.5,1.0 ; 1.0 ; 10.0)$ for time intervals $\tau=0.5,1$, respectively.
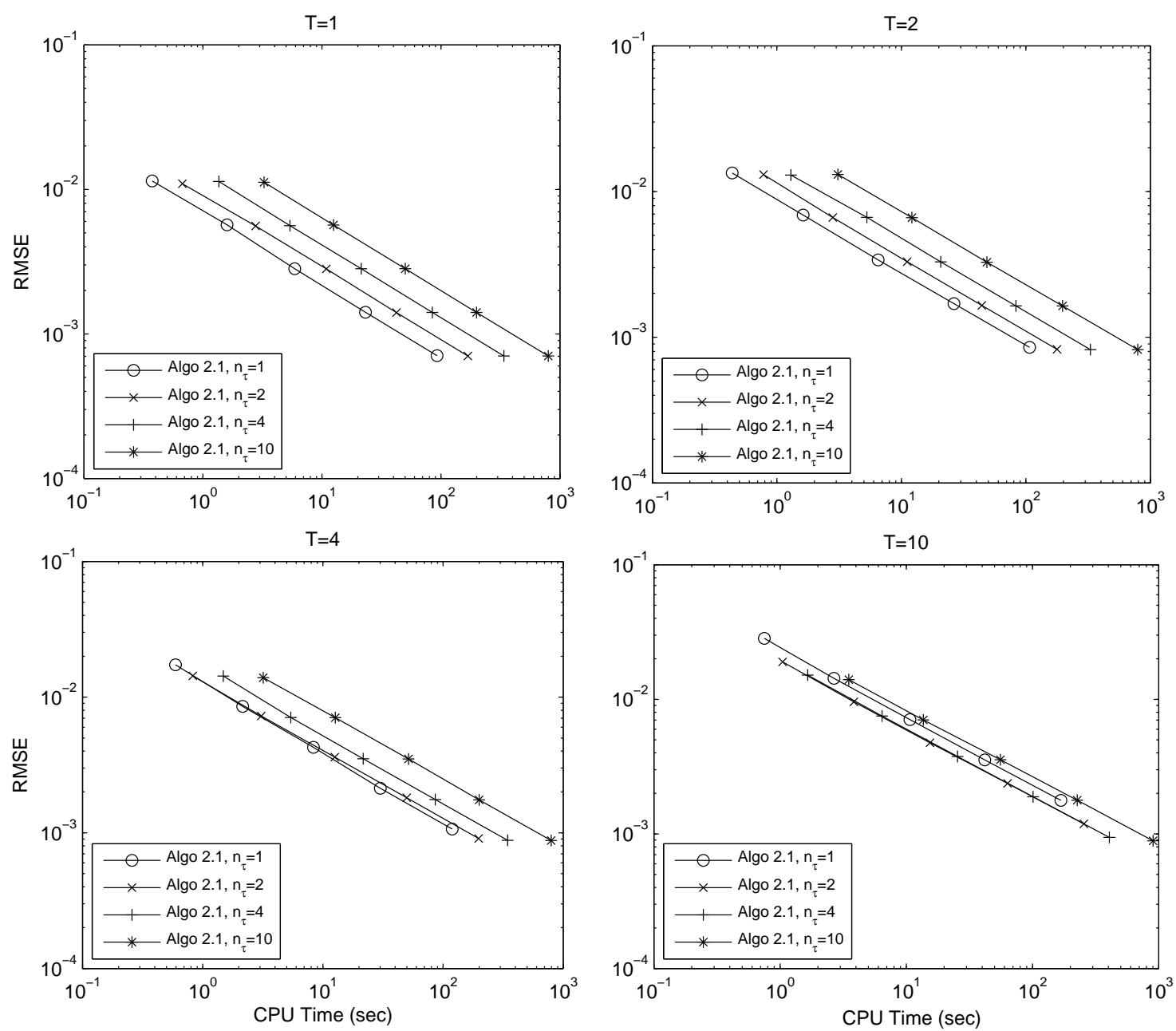

Figure 4: Convergence analysis of Algorithm 3.1 for the $\Gamma$-OU process $X_{t}$ based on the parameters $\left(\delta, \rho ; \theta ; X_{0}\right)=(0.5,1.0 ; 1.0 ; 10.0)$ for $T=1,2,4,10$ and $n_{\tau}=1,2,3,4,10$, respectively. 

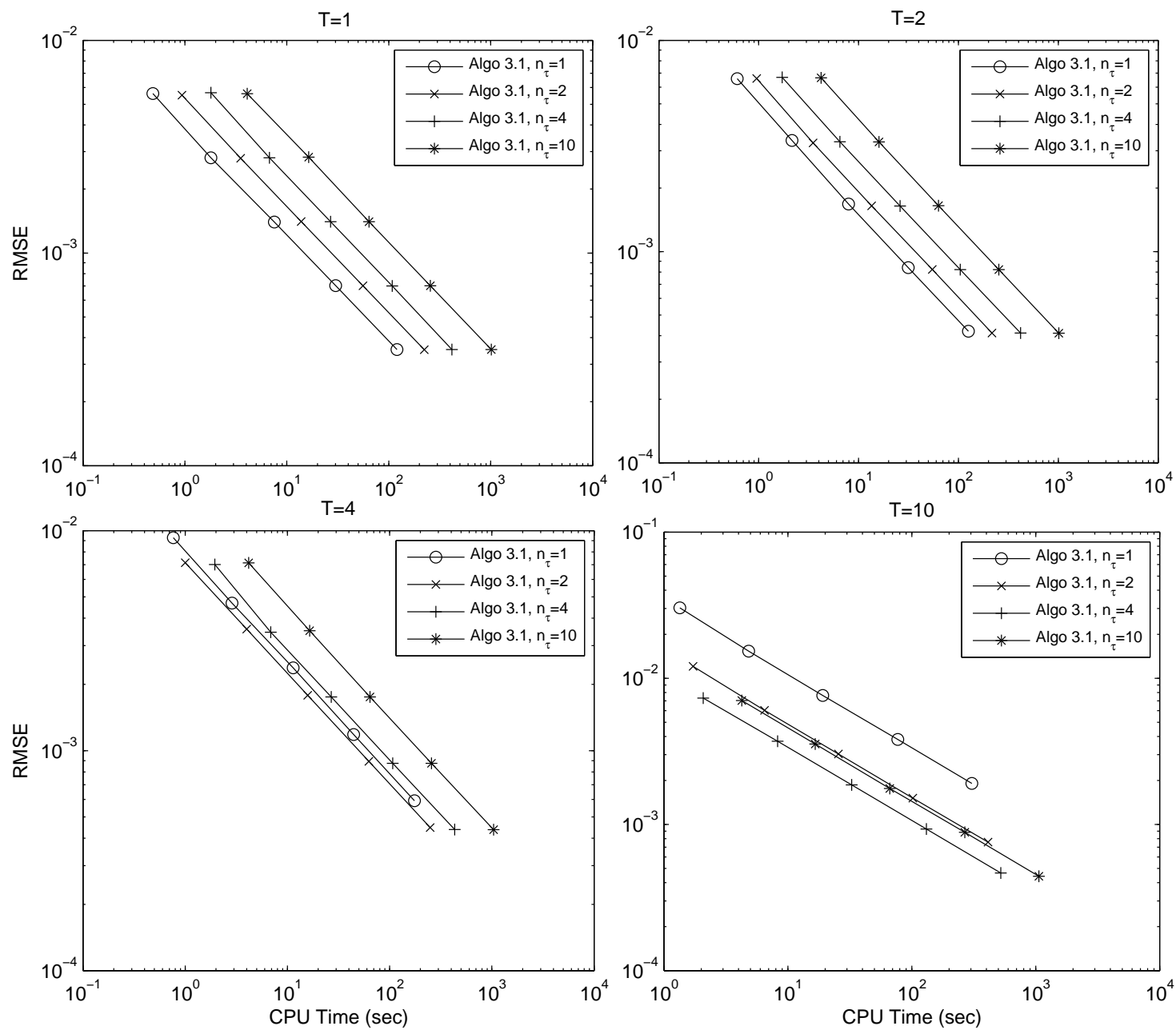

Figure 5: Convergence analysis of Algorithm 4.1 for the OU- $\Gamma$ process $Y_{t}$ based on the parameters $\left(\delta, \varrho ; \alpha, \beta ; Y_{0}\right)=(0.5,1.0 ; 2.0,2.0 ; 10.0)$ for $T=1,2,4,10$ and $n_{\tau}=1,2,3,4,10$, respectively. 
Table 2: Comparison between the true means (5.1) and the associated simulation results using Algorithm 3.1 for the $\Gamma$-OU process based on the parameters $\left(\delta, \rho ; \theta ; X_{0}\right)=(0.5,1.0 ; 1.0 ; 10.0)$ for $T=$ $1,2,4,10$ and $n_{\tau}=1,2,4,10$, respectively, with the associated plots provided in Figure 4.

\begin{tabular}{|c|c|c|c|c|c|c|c|c|c|c|c|c|}
\hline Period $T$ & Paths & True & Simulation & Error & Error \% & RMSE & Time & Simulation & Error & Error \% & RMSE & Time \\
\hline & & & \multicolumn{5}{|c|}{$n_{\tau}=1$} & \multicolumn{5}{|c|}{$n_{\tau}=2$} \\
\hline \multirow[t]{6}{*}{$T=1$} & 10,000 & 6.8522 & 6.8418 & -0.0104 & $-0.15 \%$ & 0.0114 & 0.38 & 6.8276 & -0.0247 & $-0.36 \%$ & 0.0109 & 0.67 \\
\hline & 40,000 & 6.8522 & 6.8543 & 0.0021 & $0.03 \%$ & 0.0057 & 1.59 & 6.8507 & -0.0015 & $-0.02 \%$ & 0.0056 & 2.77 \\
\hline & 160,000 & 6.8522 & 6.8536 & 0.0014 & $0.02 \%$ & 0.0028 & 5.91 & 6.8520 & -0.0003 & $0.00 \%$ & 0.0028 & 10.89 \\
\hline & 640,000 & 6.8522 & 6.8515 & -0.0007 & $-0.01 \%$ & 0.0014 & 23.19 & 6.8510 & -0.0013 & $-0.02 \%$ & 0.0014 & 42.28 \\
\hline & $2,560,000$ & 6.8522 & 6.8522 & -0.0000 & $0.00 \%$ & 0.0007 & 92.81 & 6.8516 & -0.0006 & $-0.01 \%$ & 0.0007 & 168.63 \\
\hline & & & \multicolumn{5}{|c|}{$n_{\tau}=4$} & \multicolumn{5}{|c|}{$n_{\tau}=10$} \\
\hline \multirow[t]{6}{*}{$T=1$} & 10,000 & 6.8522 & 6.8512 & -0.0011 & $-0.02 \%$ & 0.0113 & 1.36 & 6.8600 & 0.0078 & $0.11 \%$ & 0.0112 & 3.27 \\
\hline & 40,000 & 6.8522 & 6.8532 & 0.0010 & $0.01 \%$ & 0.0056 & 5.39 & 6.8549 & 0.0026 & $0.04 \%$ & 0.0057 & 12.52 \\
\hline & 160,000 & 6.8522 & 6.8562 & 0.0040 & $0.06 \%$ & 0.0028 & 21.41 & 6.8558 & 0.0036 & $0.05 \%$ & 0.0028 & 50.13 \\
\hline & 640,000 & 6.8522 & 6.8505 & -0.0017 & $-0.02 \%$ & 0.0014 & 84.63 & 6.8523 & 0.0001 & $0.00 \%$ & 0.0014 & 199.38 \\
\hline & $2,560,000$ & 6.8522 & 6.8524 & 0.0002 & $0.00 \%$ & 0.0007 & 338.53 & 6.8521 & -0.0001 & $0.00 \%$ & 0.0007 & 798.98 \\
\hline & & & \multicolumn{5}{|c|}{$n_{\tau}=1$} & \multicolumn{5}{|c|}{$n_{\tau}=2$} \\
\hline \multirow[t]{6}{*}{$T=2$} & 10,000 & 4.9430 & 4.9463 & 0.0033 & $0.07 \%$ & 0.0134 & 0.44 & 4.9224 & -0.0207 & $-0.42 \%$ & 0.0131 & 0.78 \\
\hline & 40,000 & 4.9430 & 4.9475 & 0.0045 & $0.09 \%$ & 0.0069 & 1.63 & 4.9423 & -0.0007 & $-0.01 \%$ & 0.0066 & 2.81 \\
\hline & 160,000 & 4.9430 & 4.9395 & -0.0036 & $-0.07 \%$ & 0.0034 & 6.52 & 4.9412 & -0.0018 & $-0.04 \%$ & 0.0033 & 11.14 \\
\hline & 640,000 & 4.9430 & 4.9414 & -0.0016 & $-0.03 \%$ & 0.0017 & 26.47 & 4.9422 & -0.0008 & $-0.02 \%$ & 0.0017 & 44.33 \\
\hline & $2,560,000$ & 4.9430 & 4.9433 & 0.0003 & $0.01 \%$ & 0.0009 & 107.13 & 4.9442 & 0.0012 & $0.02 \%$ & 0.0008 & 177.84 \\
\hline & & & \multicolumn{5}{|c|}{$n_{\tau}=4$} & \multicolumn{5}{|c|}{$n_{\tau}=10$} \\
\hline \multirow[t]{6}{*}{$T=2$} & 10,000 & 4.9430 & 4.9315 & -0.0116 & $-0.23 \%$ & 0.0130 & 1.30 & 4.9402 & -0.0028 & $-0.06 \%$ & 0.0131 & 3.09 \\
\hline & 40,000 & 4.9430 & 4.9533 & 0.0103 & $0.21 \%$ & 0.0066 & 5.28 & 4.9533 & 0.0103 & $0.21 \%$ & 0.0066 & 12.16 \\
\hline & 160,000 & 4.9430 & 4.9421 & -0.0010 & $-0.02 \%$ & 0.0033 & 20.69 & 4.9402 & -0.0029 & $-0.06 \%$ & 0.0033 & 48.67 \\
\hline & 640,000 & 4.9430 & 4.9416 & -0.0015 & $-0.03 \%$ & 0.0016 & 83.19 & 4.9404 & -0.0027 & $-0.05 \%$ & 0.0016 & 196.84 \\
\hline & $2,560,000$ & 4.9430 & 4.9433 & 0.0003 & $0.01 \%$ & 0.0008 & 329.92 & 4.9424 & -0.0006 & $-0.01 \%$ & 0.0008 & 792.38 \\
\hline & & & \multicolumn{5}{|c|}{$n_{\tau}=1$} & \multicolumn{5}{|c|}{$n_{\tau}=2$} \\
\hline \multirow[t]{6}{*}{$T=4$} & 10,000 & 3.0827 & 3.1051 & 0.0225 & $0.73 \%$ & 0.0173 & 0.59 & 3.1056 & 0.0229 & $0.74 \%$ & 0.0143 & 0.83 \\
\hline & 40,000 & 3.0827 & 3.0883 & 0.0056 & $0.18 \%$ & 0.0085 & 2.16 & 3.0780 & -0.0047 & $-0.15 \%$ & 0.0073 & 3.08 \\
\hline & 160,000 & 3.0827 & 3.0846 & 0.0019 & $0.06 \%$ & 0.0043 & 8.36 & 3.0796 & -0.0031 & $-0.10 \%$ & 0.0036 & 12.58 \\
\hline & 640,000 & 3.0827 & 3.0855 & 0.0028 & $0.09 \%$ & 0.0021 & 30.08 & 3.0819 & -0.0007 & $-0.02 \%$ & 0.0018 & 49.94 \\
\hline & $2,560,000$ & 3.0827 & 3.0833 & 0.0006 & $0.02 \%$ & 0.0011 & 119.14 & 3.0826 & -0.0001 & $0.00 \%$ & 0.0009 & 198.17 \\
\hline & & & \multicolumn{5}{|c|}{$n_{\tau}=4$} & \multicolumn{5}{|c|}{$n_{\tau}=10$} \\
\hline \multirow[t]{6}{*}{$T=4$} & 10,000 & 3.0827 & 3.1114 & 0.0287 & $0.93 \%$ & 0.0143 & 1.48 & 3.0842 & 0.0015 & $0.05 \%$ & 0.0139 & 3.19 \\
\hline & 40,000 & 3.0827 & 3.0790 & -0.0037 & $-0.12 \%$ & 0.0071 & 5.41 & 3.0863 & 0.0037 & $0.12 \%$ & 0.0071 & 12.72 \\
\hline & 160,000 & 3.0827 & 3.0832 & 0.0005 & $0.02 \%$ & 0.0035 & 21.64 & 3.0843 & 0.0016 & $0.05 \%$ & 0.0035 & 51.70 \\
\hline & 640,000 & 3.0827 & 3.0821 & -0.0005 & $-0.02 \%$ & 0.0018 & 86.30 & 3.0853 & 0.0026 & $0.08 \%$ & 0.0018 & 199.48 \\
\hline & $2,560,000$ & 3.0827 & 3.0803 & -0.0024 & $-0.08 \%$ & 0.0009 & 345.45 & 3.0831 & 0.0004 & $0.01 \%$ & 0.0009 & 795.36 \\
\hline & & & & & $n_{\tau}=1$ & & & & & $n_{\tau}=2$ & & \\
\hline$T=10$ & 10,000 & 2.0539 & 2.0444 & -0.0095 & $-0.46 \%$ & 0.0284 & 0.75 & 2.0339 & -0.0200 & $-0.97 \%$ & 0.0190 & 1.05 \\
\hline & 40,000 & 2.0539 & 2.0542 & 0.0003 & $0.02 \%$ & 0.0143 & 2.67 & 2.0646 & 0.0107 & $0.52 \%$ & 0.0096 & 3.84 \\
\hline & 160,000 & 2.0539 & 2.0473 & -0.0066 & $-0.32 \%$ & 0.0071 & 10.69 & 2.0550 & 0.0011 & $0.05 \%$ & 0.0048 & 15.44 \\
\hline & 640,000 & 2.0539 & 552 & 0.0013 & $0.06 \%$ & 0.0035 & 41.84 & 2.0556 & 0.0017 & $0.08 \%$ & 0.0024 & 63.53 \\
\hline & $2,560,000$ & 2.0539 & 2.0542 & 0.0003 & $0.01 \%$ & 0.0018 & 168.27 & 2.0550 & 0.0011 & $0.05 \%$ & 0.0012 & 255.75 \\
\hline & & & & & $n_{\tau}=4$ & & & & & $n_{\tau}=10$ & & \\
\hline$T=10$ & 10,000 & 2.0539 & 2.0415 & -0.0124 & $-0.60 \%$ & 0.0151 & 1.66 & 2.0366 & -0.0173 & $-0.84 \%$ & 0.0140 & 3.50 \\
\hline & 40,000 & 2.0539 & 2.0538 & -0.0001 & $-0.01 \%$ & 0.0075 & 6.44 & 2.0446 & -0.0093 & $-0.45 \%$ & 0.0070 & 13.66 \\
\hline & 160,000 & 2.0539 & 2.0520 & -0.0019 & $-0.09 \%$ & 0.0038 & 25.45 & 2.0596 & 0.0056 & $0.28 \%$ & 0.0036 & 55.66 \\
\hline & 640,000 & 2.0539 & 2.0554 & 0.0015 & $0.07 \%$ & 0.0019 & 101.14 & 2.0548 & 0.0009 & $0.04 \%$ & 0.0018 & 226.19 \\
\hline & $2,560,000$ & 2.0539 & 2.0515 & -0.0024 & $-0.12 \%$ & 0.0009 & 407.13 & 2.0539 & 0.0000 & $0.00 \%$ & 0.0009 & 902.73 \\
\hline
\end{tabular}


Table 3: Comparison between the true means (5.2) and the associated simulation results using Algorithm 4.1 for the OU- $\Gamma$ process based on the parameters $\left(\delta, \varrho ; \alpha, \beta ; X_{0}\right)=(0.5,1.0 ; 2.0,2.0 ; 10.0)$ for $T=1,2,4,10$ and $n_{\tau}=1,2,4,10$, respectively, with the associated plots provided in Figure 5.

\begin{tabular}{|c|c|c|c|c|c|c|c|c|c|c|c|c|}
\hline Period $T$ & Paths & True & Simulation & Error & Error \% & RMSE & Time & Simulation & Error & Error \% & RMSE & Time \\
\hline & & & \multicolumn{5}{|c|}{$n_{\tau}=1$} & \multicolumn{5}{|c|}{$n_{\tau}=2$} \\
\hline \multirow[t]{6}{*}{$T=1$} & 10,000 & 6.8522 & 6.8471 & -0.0051 & $-0.07 \%$ & 0.0056 & 0.48 & 6.8538 & 0.0016 & $0.02 \%$ & 0.0055 & 0.94 \\
\hline & 40,000 & 6.8522 & 6.8527 & 0.0005 & $0.01 \%$ & 0.0028 & 1.80 & 6.8535 & 0.0012 & $0.02 \%$ & 0.0028 & 3.53 \\
\hline & 160,000 & 6.8522 & 6.8500 & -0.0022 & $-0.03 \%$ & 0.0014 & 7.53 & 6.8518 & -0.0004 & $-0.01 \%$ & 0.0014 & 13.81 \\
\hline & 640,000 & 6.8522 & 6.8522 & -0.0001 & $0.00 \%$ & 0.0007 & 30.14 & 6.8517 & -0.0006 & $-0.01 \%$ & 0.0007 & 55.92 \\
\hline & $2,560,000$ & 6.8522 & 6.8521 & -0.0001 & $0.00 \%$ & 0.0004 & 120.41 & 6.8521 & -0.0001 & $0.00 \%$ & 0.0004 & 223.25 \\
\hline & & & \multicolumn{5}{|c|}{$n_{\tau}=4$} & \multicolumn{5}{|c|}{$n_{\tau}=10$} \\
\hline \multirow[t]{6}{*}{$T=1$} & 10,000 & 6.8522 & 6.8552 & 0.0029 & $0.04 \%$ & 0.0057 & 1.80 & 6.8530 & 0.0007 & $0.01 \%$ & 0.0056 & 4.09 \\
\hline & 40,000 & 6.8522 & 6.8521 & -0.0001 & $0.00 \%$ & 0.0028 & 6.75 & 6.8577 & 0.0055 & $0.08 \%$ & 0.0028 & 16.39 \\
\hline & 160,000 & 6.8522 & 6.8527 & 0.0005 & $0.01 \%$ & 0.0014 & 26.92 & 6.8514 & -0.0008 & $-0.01 \%$ & 0.0014 & 63.95 \\
\hline & 640,000 & 6.8522 & 6.8508 & -0.0014 & $-0.02 \%$ & 0.0007 & 108.23 & 6.8524 & 0.0002 & $0.00 \%$ & 0.0007 & 256.14 \\
\hline & $2,560,000$ & 6.8522 & 6.8525 & 0.0003 & $0.00 \%$ & 0.0004 & 417.30 & 6.8523 & 0.0001 & $0.00 \%$ & 0.0004 & $1,016.22$ \\
\hline & & & \multicolumn{5}{|c|}{$n_{\tau}=1$} & \multicolumn{5}{|c|}{$n_{\tau}=2$} \\
\hline \multirow[t]{6}{*}{$T=2$} & 10,000 & 4.9430 & 4.9457 & 0.0026 & $0.05 \%$ & 0.0066 & 0.61 & 4.9458 & 0.0028 & $0.06 \%$ & 0.0066 & 0.95 \\
\hline & 40,000 & 4.9430 & 4.9422 & -0.0008 & $-0.02 \%$ & 0.0034 & 2.16 & 4.9408 & -0.0023 & $-0.05 \%$ & 0.0033 & 3.50 \\
\hline & 160,000 & 4.9430 & 4.9424 & -0.0007 & $-0.01 \%$ & 0.0017 & 7.94 & 4.9426 & -0.0005 & $-0.01 \%$ & 0.0016 & 13.53 \\
\hline & 640,000 & 4.9430 & 4.9432 & 0.0001 & $0.00 \%$ & 0.0008 & 31.44 & 4.9423 & -0.0008 & $-0.02 \%$ & 0.0008 & 54.81 \\
\hline & $2,560,000$ & 4.9430 & 4.9433 & 0.0003 & $0.01 \%$ & 0.0004 & 126.03 & 4.9431 & 0.0001 & $0.00 \%$ & 0.0004 & 216.33 \\
\hline & & & \multicolumn{5}{|c|}{$n_{\tau}=4$} & \multicolumn{5}{|c|}{$n_{\tau}=10$} \\
\hline \multirow[t]{6}{*}{$T=2$} & 10,000 & 4.9430 & 4.9498 & 0.0067 & $0.14 \%$ & 0.0067 & 1.72 & 4.9424 & -0.0007 & $-0.01 \%$ & 0.0066 & 4.22 \\
\hline & 40,000 & 4.9430 & 4.9469 & 0.0038 & $0.08 \%$ & 0.0033 & 6.52 & 4.9472 & 0.0042 & $0.08 \%$ & 0.0033 & 16.03 \\
\hline & 160,000 & 4.9430 & 4.9439 & 0.0009 & $0.02 \%$ & 0.0016 & 26.08 & 4.9459 & 0.0029 & $0.06 \%$ & 0.0016 & 63.27 \\
\hline & 640,000 & 4.9430 & 4.9427 & -0.0004 & $-0.01 \%$ & 0.0008 & 104.27 & 4.9435 & 0.0005 & $0.01 \%$ & 0.0008 & 253.89 \\
\hline & $2,560,000$ & 4.9430 & 4.9432 & 0.0002 & $0.00 \%$ & 0.0004 & 418.30 & 4.9428 & -0.0002 & $0.00 \%$ & 0.0004 & $1,013.73$ \\
\hline & & & \multicolumn{5}{|c|}{$n_{\tau}=1$} & \multicolumn{5}{|c|}{$n_{\tau}=2$} \\
\hline \multirow[t]{6}{*}{$T=4$} & 10,000 & 3.0827 & 3.0826 & -0.0000 & $0.00 \%$ & 0.0093 & 0.77 & 3.0942 & 0.0115 & $0.37 \%$ & 0.0071 & 1.00 \\
\hline & 40,000 & 3.0827 & 3.0826 & -0.0001 & $0.00 \%$ & 0.0047 & 2.88 & 3.0835 & 0.0008 & $0.03 \%$ & 0.0036 & 4.00 \\
\hline & 160,000 & 3.0827 & 3.0854 & 0.0027 & $0.09 \%$ & 0.0024 & 11.38 & 3.0835 & 0.0008 & $0.03 \%$ & 0.0018 & 15.81 \\
\hline & 640,000 & 3.0827 & 3.0812 & -0.0015 & $-0.05 \%$ & 0.0012 & 44.47 & 3.0823 & -0.0004 & $-0.01 \%$ & 0.0009 & 62.72 \\
\hline & $2,560,000$ & 3.0827 & 3.0836 & 0.0010 & $0.03 \%$ & 0.0006 & 175.28 & 3.0830 & 0.0003 & $0.01 \%$ & 0.0004 & 249.16 \\
\hline & & & \multicolumn{5}{|c|}{$n_{\tau}=4$} & \multicolumn{5}{|c|}{$n_{\tau}=10$} \\
\hline \multirow[t]{6}{*}{$T=4$} & 10,000 & 3.0827 & 3.0794 & -0.0033 & $-0.11 \%$ & 0.0070 & 1.95 & 3.0862 & 0.0035 & $0.11 \%$ & 0.0071 & 4.17 \\
\hline & 40,000 & 3.0827 & 3.0813 & -0.0014 & $-0.05 \%$ & 0.0035 & 6.86 & 3.0831 & 0.0004 & $0.01 \%$ & 0.0035 & 16.52 \\
\hline & 160,000 & 3.0827 & 3.0840 & 0.0013 & $0.04 \%$ & 0.0018 & 26.81 & 3.0849 & 0.0022 & $0.07 \%$ & 0.0018 & 64.23 \\
\hline & 640,000 & 3.0827 & 3.0819 & -0.0008 & $-0.02 \%$ & 0.0009 & 107.36 & 3.0822 & -0.0005 & $-0.02 \%$ & 0.0009 & 256.56 \\
\hline & $2,560,000$ & 3.0827 & 3.0823 & -0.0004 & $-0.01 \%$ & 0.0004 & 432.86 & 3.0823 & -0.0004 & $-0.01 \%$ & 0.0004 & $1,040.16$ \\
\hline & & & & & $n_{\tau}=1$ & & & & & $n_{\tau}=2$ & & \\
\hline$T=10$ & 10,000 & 2.0539 & 2.0345 & -0.0194 & $-0.94 \%$ & 0.0303 & 1.34 & 2.0523 & -0.0016 & $-0.08 \%$ & 0.0121 & 1.72 \\
\hline & 40,000 & 2.0539 & 2.0554 & 0.0015 & $0.07 \%$ & 0.0153 & 4.83 & 2.0480 & -0.0059 & $-0.29 \%$ & 0.0060 & 6.48 \\
\hline & 160,000 & 2.0539 & 2.0545 & 0.0006 & $0.03 \%$ & 0.0076 & 19.14 & 2.0550 & 0.0010 & $0.05 \%$ & 0.0030 & 25.58 \\
\hline & 640,000 & 2.0539 & 2.0561 & 0.0022 & $0.11 \%$ & 0.0038 & 77.05 & 2.0545 & 0.0006 & $0.03 \%$ & 0.0015 & 101.86 \\
\hline & $2,560,000$ & 2.0539 & 2.0527 & -0.0012 & $-0.06 \%$ & 0.0019 & 305.59 & 2.0542 & 0.0003 & $0.01 \%$ & 0.0008 & 412.20 \\
\hline & & & & & $n_{\tau}=4$ & & & & & $n_{\tau}=10$ & & \\
\hline$T=10$ & 10,000 & 2.0539 & 2.0471 & -0.0068 & $-0.33 \%$ & 0.0073 & 2.08 & 2.0526 & -0.0013 & $-0.07 \%$ & 0.0070 & 4.25 \\
\hline & 40,000 & 2.0539 & 2.0512 & -0.0027 & $-0.13 \%$ & 0.0037 & 8.28 & 2.0556 & 0.0017 & $0.08 \%$ & 0.0036 & 16.63 \\
\hline & 160,000 & 2.0539 & 2.0529 & -0.0010 & $-0.05 \%$ & 0.0019 & 32.70 & 2.0555 & 0.0016 & $0.08 \%$ & 0.0018 & 66.47 \\
\hline & 640,000 & 2.0539 & 2.0542 & 0.0003 & $0.01 \%$ & 0.0009 & 131.38 & 2.0536 & -0.0003 & $-0.02 \%$ & 0.0009 & 267.59 \\
\hline & $2,560,000$ & 2.0539 & 2.0551 & 0.0012 & $0.06 \%$ & 0.0005 & 523.14 & 2.0543 & 0.0004 & $0.02 \%$ & 0.0004 & $1,061.34$ \\
\hline
\end{tabular}




\section{Concluding Remarks}

In this paper, we have developed very efficient algorithms for the exact simulation of the $\Gamma$-OU and OU- $\Gamma$ processes based on our distributional decomposition approach. The algorithms are accurate, efficient, and have been numerically verified, with the associated performance reported in detail. To extend these models, adding an extra constant term $\mu$ as the mean-reverting (or lower bound) parameter in the Gamma-driven OU process (2.1) or (2.2) is mathematically trivial; here, we have simply set $\mu$ equal to zero to keep our paper concise. Recently, Bianchi and Fabozzi (2015) made a convincing case that the use of Lévy-based OU processes that allow only for positive jumps is obstructed by the path properties of these processes, which cannot appropriately replicate the behavior of observed CDS spreads quoted in the credit market. This drawback is mainly because they only return to stability slowly, and our simulation scheme could be extended to construct models with two or more drivers, with one of them having a fast decay rate. It is interesting to carry out the empirical work furthermore by following this line. Moreover, generalizations to time-varying parameters or dependent multivariate settings may also be possible, and in addition the associated estimation problems would be important but challenging, and we propose these as topics for future research.

\section{Acknowledgments}

The authors would like to thank the reviewer for very helpful and constructive comments and suggestions. The corresponding author Hongbiao Zhao would like to acknowledge the financial support from the National Natural Science Foundation of China (\#71401147) and the research fund provided by Shanghai University of Finance and Economics and Shanghai Institute of International Finance and Economics. 


\section{References}

Aït-Sahalia, Y. and Jacod, J. (2009). Estimating the degree of activity of jumps in high frequency data. Annals of Statistics, 37(5A):2202-2244.

Ait-Sahalia, Y. and Jacod, J. (2011). Testing whether jumps have finite or infinite activity. Annals of Statistics, 39(3):1689-1719.

Barndorff-Nielsen, O. and Shephard, N. (2001a). Modelling by Lévy processess for financial econometrics. In Barndorff-Nielsen, O., Resnick, S., and Mikosch, T., editors, Lévy Processes, pages 283-318. Birkhäuser, Boston.

Barndorff-Nielsen, O. E. (1997). Normal inverse Gaussian distributions and stochastic volatility modelling. Scandinavian Journal of Statistics, 24(1):1-13.

Barndorff-Nielsen, O. E. (1998). Processes of normal inverse Gaussian type. Finance and Stochastics, 2(1):41-68.

Barndorff-Nielsen, O. E., Jensen, J. L., and Sørensen, M. (1998). Some stationary processes in discrete and continuous time. Advances in Applied Probability, 30(4):989-1007.

Barndorff-Nielsen, O. E. and Shephard, N. (2001b). Non-Gaussian Ornstein-Uhlenbeck-based models and some of their uses in financial economics. Journal of the Royal Statistical Society: Series B (Statistical Methodology), 63(2):167-241.

Barndorff-Nielsen, O. E. and Shephard, N. (2002). Econometric analysis of realized volatility and its use in estimating stochastic volatility models. Journal of the Royal Statistical Society: Series B (Statistical Methodology), 64(2):253-280.

Barndorff-Nielsen, O. E. and Shephard, N. (2003a). Integrated OU processes and non-Gaussian OU-based stochastic volatility models. Scandinavian Journal of Statistics, 30(2):277-295.

Barndorff-Nielsen, O. E. and Shephard, N. (2003b). Realized power variation and stochastic volatility models. Bernoulli, 9(2):243-265.

Bertoin, J. (1998). Lévy Processes. Cambridge University Press.

Bertoin, J. (1999). Subordinators: Examples and applications. In Lectures on Probability Theory and Statistics, pages 1-91. Springer.

Bianchi, M. L. and Fabozzi, F. J. (2015). Investigating the performance of non-Gaussian stochastic intensity models in the calibration of credit default swap spreads. Computational Economics, 46(2):243-273.

Bianchi, M. L., Rachev, S. T., and Fabozzi, F. J. (2017). Tempered stable Ornstein-Uhlenbeck processes: A practical view. Communications in Statistics-Simulation and Computation, 46(1):423-445.

Bianchi, M. L. and Tassinari, G. L. (2018). Forward-looking portfolio selection with multivariate nonGaussian models and the Esscher transform. arXiv preprint:1805.05584. 
Broadie, M. and Kaya, Ö. (2006). Exact simulation of stochastic volatility and other affine jump diffusion processes. Operations Research, 54(2):217-231.

Brockwell, P. J., Davis, R. A., and Yang, Y. (2007). Estimation for nonnegative Lévy-driven OrnsteinUhlenbeck processes. Journal of Applied Probability, 44(4):977-989.

Cartea, Á. and Jaimungal, S. (2016). A closed-form execution strategy to target volume weighted average price. SIAM Journal on Financial Mathematics, 7(1):760-785.

Cartea, Á., Jaimungal, S., and Penalva, J. (2015). Algorithmic and High-Frequency Trading. Cambridge University Press.

Chen, N. and Huang, Z. (2013). Localization and exact simulation of Brownian motion-driven stochastic differential equations. Mathematics of Operations Research, 38(3):591-616.

Cont, R. and Tankov, P. (2004). Financial Modelling with Jump Processes. CRC Press, Boca Raton.

Creal, D. D. (2008). Analysis of filtering and smoothing algorithms for Lévy-driven stochastic volatility models. Computational Statistics \& Data Analysis, 52(6):2863-2876.

Dassios, A. and Embrechts, P. (1989). Martingales and insurance risk. Stochastic Models, 5(2):181-217.

Dassios, A. and Jang, J. (2003). Pricing of catastrophe reinsurance and derivatives using the Cox process with shot noise intensity. Finance and Stochastics, 7(1):73-95.

Dassios, A., Qu, Y., and Zhao, H. (2018). Exact simulation for a class of tempered stable and related distributions. ACM Transactions on Modeling and Computer Simulation, 28(3):20:1-20:21.

Dassios, A. and Zhao, H. (2011). A dynamic contagion process. Advances in Applied Probability, 43(3):814-846.

Dassios, A. and Zhao, H. (2013). Exact simulation of Hawkes process with exponentially decaying intensity. Electronic Communications in Probability, 18(62):1-13.

Dassios, A. and Zhao, H. (2017). Efficient simulation of clustering jumps with CIR intensity. Operations Research, 65(6):1494-1515.

Davis, M. H. (1984). Piecewise-deterministic Markov processes: A general class of non-diffusion stochastic models. Journal of the Royal Statistical Society. Series B (Methodological), 46(3):353-388.

Eberlein, E., Madan, D., Pistorius, M., and Yor, M. (2013). A simple stochastic rate model for rate equity hybrid products. Applied Mathematical Finance, 20(5):461-488.

Frühwirth-Schnatter, S. and Sögner, L. (2009). Bayesian estimation of stochastic volatility models based on OU processes with marginal Gamma law. Annals of the Institute of Statistical Mathematics, 61(1):159_ 179. 
Granelli, A. and Veraart, A. E. D. (2016). Modeling the variance risk premium of equity indices: The role of dependence and contagion. SIAM Journal on Financial Mathematics, 7(1):382-417.

Griffin, J. E. and Steel, M. F. (2006). Inference with non-Gaussian Ornstein-Uhlenbeck processes for stochastic volatility. Journal of Econometrics, 134(2):605-644.

Hainaut, D. and Devolder, P. (2008). Mortality modelling with Lévy processes. Insurance: Mathematics and Economics, 42(1):409-418.

Jongbloed, G., Van Der Meulen, F. H., and Van Der Vaart, A. W. (2005). Nonparametric inference for Lévy-driven Ornstein-Uhlenbeck processes. Bernoulli, 11(5):759-791.

Kawai, R. and Masuda, H. (2011a). Exact discrete sampling of finite variation tempered stable OrnsteinUhlenbeck processes. Monte Carlo Methods and Applications, 17(3):279-300.

Kawai, R. and Masuda, H. (2011b). On simulation of tempered stable random variates. Journal of Computational and Applied Mathematics, 235(8):2873-2887.

Kawai, R. and Masuda, H. (2012). Infinite variation tempered stable Ornstein-Uhlenbeck processes with discrete observations. Communications in Statistics-Simulation and Computation, 41(1):125-139.

Kyprianou, A. (2006). Introductory Lectures on Fluctuations of Lévy Processes with Applications. Springer, Berlin.

Madan, D., Carr, P., and Chang, E. (1998). The variance gamma process and option pricing. European Finance Review, 2(1):79-105.

Madan, D. B. and Seneta, E. (1990). The variance gamma (V.G.) model for share market returns. Journal of Business, 63(4):511-524.

Mai, H. (2014). Efficient maximum likelihood estimation for Lévy-driven Ornstein-Uhlenbeck processes. Bernoulli, 20(2):919-957.

Masuda, H. and Yoshida, N. (2005). Asymptotic expansion for Barndorff-Nielsen and Shephard's stochastic volatility model. Stochastic Processes and their Applications, 115(7):1167-1186.

Nicolato, E. and Venardos, E. (2003). Option pricing in stochastic volatility models of the OrnsteinUhlenbeck type. Mathematical Finance, 13(4):445-466.

Norberg, R. (2004). Vasiček beyond the normal. Mathematical Finance, 14(4):585-604.

Ornthanalai, C. (2014). Lévy jump risk: Evidence from options and returns. Journal of Financial Economics, 112(1):69-90.

Qu, Y., Dassios, A., and Zhao, H. (2019). Efficient simulation of Lévy-driven point processes. Advances in Applied Probability. forthcoming. 
Roberts, G. O., Papaspiliopoulos, O., and Dellaportas, P. (2004). Bayesian inference for non-Gaussian Ornstein-Uhlenbeck stochastic volatility processes. Journal of the Royal Statistical Society: Series B (Statistical Methodology), 66(2):369-393.

Sato, K.-i. (1999). Lévy Processes and Infinitely Divisible Distributions. Cambridge University Press.

Sato, K.-i. and Yamazato, M. (1984). Operator-selfdecomposable distributions as limit distributions of processes of Ornstein-Uhlenbeck type. Stochastic Processes and their Applications, 17(1):73-100.

Schoutens, W. (2003). Lévy Processes in Finance: Pricing Financial Derivatives. Wiley.

Schoutens, W. and Cariboni, J. (2010). Lévy Processes in Credit Risk. John Wiley \& Sons, Chichester.

Uhlenbeck, G. E. and Ornstein, L. S. (1930). On the theory of the Brownian motion. Physical Review, 36(5):823.

Vasicek, O. (1977). An equilibrium characterization of the term structure. Journal of Financial Economics, 5(2):177-188.

Wolfe, S. J. (1982). On a continuous analogue of the stochastic difference equation $x_{n}=\rho x_{n-1}+b_{n}$. Stochastic Processes and their Applications, 12(3):301-312.

Zhang, S. (2011). Exact simulation of tempered stable Ornstein-Uhlenbeck processes. Journal of Statistical Computation and Simulation, 81(11):1533-1544.

Zhang, S. and Zhang, X. (2008). Exact simulation of IG-OU processes. Methodology and Computing in Applied Probability, 10(3):337-355.

Zhang, S. and Zhang, X. (2009). On the transition law of tempered stable Ornstein-Uhlenbeck processes. Journal of Applied Probability, 46(3):721-731. 